\title{
Pyr3 Induces Apoptosis and Inhibits Migration in Human Glioblastoma Cells
}

\author{
Hsin-Han Chang ${ }^{a} \quad$ Yu-Chen Cheng ${ }^{b}$ Wen-Chiuan Tsaic ${ }^{c}$ Min-Jen Tsao ${ }^{d}$ \\ Ying Chen ${ }^{\mathrm{a}, \mathrm{b}}$
}

${ }^{\mathrm{a}}$ Graduate Institute of Life Science, National Defense Medical Center, Taipei, ${ }^{\mathrm{b}}$ Department of Biology and Anatomy, National Defense Medical Center, Taipei, 'Department of Pathology, Tri-Service General Hospital, National Defense Medical Center, Taipei, dDepartment of General Surgery, Zuoying Branch of Kaohsiung Armed Forces General Hospital, Taipei, Taiwan

\section{Key Words}

Pyr3 • Proliferation • Apoptosis • Migration • Glioblastoma

\begin{abstract}
Background/Aims: Glioblastoma, also known as glioblastoma multiforme (GBM), is a fastgrowing type of tumor that is the most aggressive brain malignancy in adults. According to GEO profile analysis, patients with high transient receptor potential canonical 3 (TRPC3) expression have poor survival rates. The aim of this study is to evaluate the effects of Ethyl-1(4-(2,3,3-trichloroacrylamide)phenyl)-5-(trifluoromethyl)-1H-pyrazole-4-carboxylate (Pyr3), a selective TRPC3 channel blocker, on the proliferation and migration of human glioblastoma cells. Methods: We first analyzed the TRPC3 mRNA expression in Gene Expression Omnibus (GEO) database. Then, TRPC3 protein expression was analyzed by Western blotting in three human GBM cell lines. The survival rate was measured by sulforhodamine B. JC1 staining was used to analyze the mitochondria membrane potential by flow cytometric analysis. Besides, the migration and invasion were evaluated by wound healing and Transwell assays. Annexin $V$ and 7-aminoactinomycin D staining was used to monitor the apoptosis by flow cytometric analysis. The expression of apoptotic-related and migration-related proteins after Pyr3 treatment was detected by Western blotting. In addition, an orthotropic xenograft mouse model was used to assay the effect of Pyr3 in the in vivo study. Results: Basis on the results of bioinformatics study, glioma patients with higher TRPC3 expression had a shorter survival time than those with lower TRPC3 expression. GBM cell proliferation was decreased by Pyr3 treatment. The migration and invasion abilities of glioma cells were also inhibited via focal adhesion kinase and myosin light chain dephosphorization after Pyr3 treatment. Moreover, Pyr3 induced caspase-dependent apoptosis and mitochondria membrane potential imbalance in the GBM cells. In a xenograft animal model, Pyr3 in combination with temozolomide (TMZ) inhibited GBM tumor growth. Conclusion: Pyr3 inhibited GBM tumor growth in vitro and in vivo. Pyr3-TMZ combination therapy could be used to treat glioblastoma in the future.

Hsin-Han Chang and Yu-Chen Cheng contributed equally to this work.




\section{Cellular Physiology Cell Physiol Biochem 2018;48:1694-1702 \begin{tabular}{l|l} 
and Biochemistry POI: 10.1159/000492293 & $\begin{array}{l}\text { (c) } 2018 \text { The Author(s). Published by S. Karger AG, Basel } \\
\text { www.karger.com/cpb }\end{array}$
\end{tabular} \\ Chablished online: August al: Pyr3 Inhibits the Progression of Human Glioblastoma Cells}

\section{Introduction}

The World Health Organization (WHO) classifies gliomas, which are derived from the glial cells of the brain, into four grades according to their pathological organization [1]. Grade IV stellate cell tumors are called glioblastoma multiforme (GBM); GBM tumors are the most aggressive and the most common neuroblastomas [2]. Current treatments for GBM include surgical resection, radiotherapy and chemotherapy. However, GBM cells are integrated with the surrounding normal brain tissue, resulting in poor surgical reaction and causing the GBM cells to spread easily and relapse [1]. Chemotherapy increases patient survival rates, but chemotherapy drugs may not easily pass through the blood-brain barrier (BBB). Therefore, most patients have poor two-year survival rates, even with treatment $[3,4]$. An effective drug for treating GBM is necessary.

Transient receptor potential channel 3 (TRPC3) is a cationic channel that is permeable to calcium ions and regulates intracellular calcium-related signaling pathways by activating $\mathrm{G}$ protein-coupled receptors [5]. In breast cancer, high TRPC3 expression levels are correlated with increased metastatic behavior [6]. High expression levels of TRPC3 have also been reported in ovarian cancer [7]. In melanoma cells, TRPC3 inhibition reduces migration and proliferation [5]. The TRPC1 channel inhibitor 2-APB reduces migration in the grade IV glioma cell line D54MG [8]. According to Gene Expression Omnibus (GE0) data, high-grade glioma patients have higher TRPC3 expression levels than those of control subjects. Reduced proliferation was also recently reported in TRPC $3^{\text {Low }}$ U87MG cells [9]. However, the role of TRPC3 in migration and invasion and its underlying mechanism in GBM cells remain unclear. The aim of this study is to determine the effects of Ethyl-1-(4-(2, 3,3-trichloroacrylamide) phenyl)-5-(trifluoromethyl)-1H-pyrazole-4-carboxylate (Pyr3), a TRPC3 inhibitor, on survival, migration and invasion in GBM cells.

\section{Materials and Methods}

\section{Analysis of human glioma datasets from the GEO database}

The genomic datasets obtained from the GEO database were analyzed with methodology developed by Dr. Hueng and colleagues. Briefly, 100 sets of de-linked data (GDS4465/210814_at) on TRPC3 mRNA expression, age, pathologic grading of primary gliomas and overall survival time were obtained from https://www.ncbi.nlm.nih.gov/geo/tools/profileGraph.cgi?ID=GDS4465:210814_at. A total of 26 datasets were used for the statistical analyses.

\section{Cell culture}

Human LN229 and U87 MG glioblastoma cell lines were provided by Dr. Hueng of the National Defense Medical Center, Taiwan. LN229-Luc2 cells were derived from a stable transfection of pLuc2-iRFP and were selected with a BD FACSAria sorter with BD FACSDiva software (BDBiosciences). The cells were grown in Dulbecco's Modified Eagle's Medium (DMEM) containing 10\% fetal bovine serum and $100 \mathrm{IU} / \mathrm{ml}$ penicillin and streptomycin (pH 7.4) (all obtained from Gibco) in a humidified atmosphere of $5 \% \mathrm{CO}_{2}$ at $37{ }^{\circ} \mathrm{C}$.

\section{Drugs}

Pyr3 was purchased from Cayman Chemical. Temozolomide (TMZ) was purchased from MedChem Express (MCE).

\section{Cell survival assay}

LN229 and U87 MG glioblastoma cells were cultured at $1 \times 10^{5}$ cells per well in a 24-well plate. Different concentrations of Pyr3 or a vehicle control were then added to the culture medium for 24 hours. After drug treatment, the culture medium was removed, and $50 \mu \mathrm{L}$ of $10 \%$ trichloroacetic acid (TCA) (Sigma) in PBS was added and incubated at $4{ }^{\circ} \mathrm{C}$ for 30 mins. The TCA buffer was removed, and the cells were washed with 


\section{Cellular Physiology Cell Physiol Biochem 2018;48:1694-1702 and Biochemistry \begin{tabular}{c|c} 
DOI: 10.1159/000492293 \\
Published online: August 2, 2018 & $\begin{array}{l}\text { O 2018 The Author(s). Published by S. Karger AG, Basel } \\
\text { www.karger.com/cpb }\end{array}$
\end{tabular} \\ Chang et al.: Pyr3 Inhibits the Progression of Human Glioblastoma Cells}

$\mathrm{ddH}_{2} \mathrm{O} 5$ times and then air dried. Next, $500 \mu \mathrm{L}$ of $0.4 \%(\mathrm{w} / \mathrm{v})$ sulforhodamine B (SRB) in $1 \%$ acetic acid was added for $20 \mathrm{~min}$ at room temperature. Each well was washed with $1 \%$ acetic acid 5 times and then air dried. Finally, $500 \mu \mathrm{L}$ of $10 \mathrm{mM}$ Tris ( $\mathrm{pH}=10.5)$ was added to each well, and the absorbance at $560 \mathrm{~nm}$ was measured by an ELISA reader.

\section{Cell migration assays}

Cell migration ability was assayed via wound healing and Transwell assays. Wound healing migration assays were conducted by seeding $1 \times 10^{6}$ U87 MG or LN229 glioma cells to form a monolayer in 3.5-cm culture dishes. The cells were then cultured for $16 \mathrm{~h}$ after being scratched with a P200 pipette tip and photographed; the migration assay results are representative of three different experiments and were analyzed with ImageJ. Transwell migration assays were conducted by seeding $8 \times 10^{4}$ LN229 or U87 MG glioma cells in the upper chamber of a Transwell plate (Costar). After incubation at $37^{\circ} \mathrm{C}$ for $16 \mathrm{hrs}$, the cells in the lower chamber were fixed with formalin and stained with Coomassie Brilliant Blue G250 solution (Sigma). The migrated cells were counted for three randomly selected fields from each membrane three times per group for each experiment.

\section{Cell invasion assay}

Transwell invasion assays were conducted by seeding $4 \times 10^{4}$ U87MG or LN229 glioma cells in the upper chamber of a Transwell plate. Before cell seeding, $0.5 \%$ Matrigel in a coating buffer solution (BD) was added to the upper chamber and then incubated at $37^{\circ} \mathrm{C}$ for $16 \mathrm{hrs}$. The cells in the bottom chamber were fixed with formalin and stained with Coomassie Brilliant Blue G250 solution. The invaded cells were counted for three randomly selected fields from each membrane three times per group for each experiment.

\section{Flow cytometric analysis}

Cell cycle and apoptosis assays and mitochondrial membrane potential ratio assays were conducted by seeding $5 \times 10^{5}$ LN229 or U87 MG glioma cells in 6-well plates. After cell attachment, the drug treatments were applied for 96 hours. For the cell cycle assays, the cells were fixed and stained with PI solution. For the apoptosis assays, Annexin V-phycoerythrin (PE) and 7-aminoactinomycin D (7-AAD) were purchased from Becton Dickinson; the cells were harvested and processed for Annexin V staining according to the manufacturer's instructions (Biovision). The cells were then washed with binding buffer [4-(2-hydroxyethyl)-1-piperazineethanesulfonic acid, $140 \mathrm{mmol} / \mathrm{L} \mathrm{NaCl}$, and $5 \mathrm{mmol} / \mathrm{L} \mathrm{CaCl}_{2}$ at $\mathrm{pH}$ 7.4], stained with anti-Annexin $\mathrm{V}$ antibody and then counterstained with PI at room temperature. For the mitochondrial membrane potential ratio assays, the cells were harvested and measured using a flow cytometry mitochondrial membrane potential detection kit (JC1 staining, Mito Screen, BD Biosciences). The results were measured using a FACSVerse laser flow cytometric analysis system (Becton Dickinson). Ten thousand cells were analyzed for each sample.

\section{Caspase-3/7 analysis}

Caspase-GLO 3/7 assay kits (Promega) were used for caspase-3/7 analyses according to the manufacturer's instructions. The cells were treated as described for the cell survival assays. After drug treatment, the cells were lysed with reagent, and the contents of the wells were gently mixed using a plate shaker at 300-500 rpm for 30 seconds; then, the plates were incubated at room temperature for 30 minutes. The results were measured using a luminometer.

\section{Western blotting}

After various treatments, glioma cells were homogenized in lysis buffer $\left(10 \mathrm{mM}\right.$ EGTA, $2 \mathrm{mM} \mathrm{MgCl} \mathrm{m}_{2}$ $60 \mathrm{mM}$ PIPES, $25 \mathrm{mM}$ HEPES, 0.15\% Triton X-100, $1 \mu \mathrm{g} / \mathrm{mL}$ pepstatin A, $1 \mu \mathrm{g} / \mathrm{mL}$ leupeptin, $1 \mathrm{mM} \mathrm{NaF}$, and $1 \mathrm{mM}$ phenylmethylsulfonyl fluoride). Protein samples (50 $\mu \mathrm{g}$ per lane) were electrophoresed on a $10 \%$ SDS polyacrylamide gel and then transferred onto a nitrocellulose membrane (Bio-Rad). Strips from the membrane were blocked with $5 \%$ non-fat milk in Tris-buffered saline, $\mathrm{pH} 7.4$, containing $0.1 \%$ Tween (TBS-Tween) and were incubated overnight at $4{ }^{\circ} \mathrm{C}$ with 1:500 dilutions of mouse antibodies against phosphorylated FAK (p-FAK) or FAK (Becton Dickinson) or rabbit antibodies against phosphorylated MLC (p-MLC), MLC (Abcam), PARP, cleaved caspase 3, cleaved caspase 7 or GAPDH (Cell Signaling Technology). After being washed, the membrane strips were incubated with 1:5000 dilutions of HRP-conjugated 


\section{Cellular Physiology Cell Physiol Biochem 2018;48:1694-1702 \begin{tabular}{l|l|l} 
DOI: 10.1159/000492293 2018 & $\begin{array}{l}\text { O } 2018 \text { The Author(s). Published by S. Karger AG, Basel } \\
\text { www.karger.com/cpb }\end{array}$ \\
and Biochemistry Published online: August 2,
\end{tabular}}

anti-rabbit or anti-mouse IgG antibodies (Promega). Next, the blots were treated with an ECL substrate developing solution (Bio-Rad). The densities of the bands on the nitrocellulose membranes were quantified by Image Lab 6.0 (Bio-Rad); the density of the control sample was set as $100 \%$, and the density of the test sample was expressed relative to the expression of the internal control. Phosphorylated protein levels were first normalized to the total protein levels.

\section{Animal xenograft model}

All mouse experiments were approved by the Laboratory Animal Center of the National Defense Medical Center, Taiwan (IACUC No. 16-275). BALB/cAnN.Cg-Foxn1nu/CrlNarl nude mice (20-25 g) were anesthetized with an $\mathrm{O}_{2}$ /isoflurane mixture. Next, $10^{5}$ control or LN229-Luc2 cells were implanted into the right cerebral hemisphere of the mice. Four days after implantation, the mice were assigned to four groups that received vehicle control (in 10\% Tween 80, n=5), TMZ (n=3), Pyr3 (n=5) or Pyr3+TMZ (n=5) treatment. Pyr3 in 10\% Tween 80 was administered via i.p. injection at $20 \mathrm{mg} / \mathrm{kg} /$ day for 7 days. TMZ was administered via oral gavage of $5 \mathrm{mg} / \mathrm{kg} /$ day for 7 days. Animals were euthanized using $\mathrm{CO}_{2}$ after 12 days; the brains were fixed in formalin, embedded in paraffin, and cut into serial sections. The bioluminescence intensity of the implanted tumors was monitored with a non-invasive In vivo Imaging System (IVIS) three times per week. The body weights of the mice were also measured three times per week.

\section{Statistical analysis}

The overall survival of the GEO datasets (GDS4465/210814_at /TRPC3) was analyzed by the KaplanMeier method. A one-tailed test was used to calculate the TRPC3 expression levels for the groups of WHO pathologic grade gliomas. Cohorts of low versus high TRPC3 gene expression levels were established for both WHO grade III and grade IV human glioma groups. The cut-off value for TRPC3 expression was based on the conditional inference tree via the 'party' package with R language (R 3.1.2 software). All experiments were performed at least three times, and the results are expressed as the means \pm SEM for all experiments. Differences between the means were assessed using the Kruskal-Wallis test. The Mann-Whitney test was used for post hoc analyses. Statistical significance was set at $\mathrm{p}<0.05$.

\section{Results}

\section{TRPC3 is correlated with survival rates in GBM patients and cell lines}

According to the GEO profile analysis, GBM patients with low TRPC3 expression have longer survival times than those with high expression (Fig. 1A). The median survival rate in the low TRPC3 expression group is 3.2-fold longer than that in the high TRPC3 expression group (Fig. 1A). Thus, TRPC3 expression levels are correlated with GBM patient survival. In addition, TRPC3 expression levels in GBM cells were higher than those in normal human astrocytes (HAs) (Fig. 1B). To further identify the function of TRPC3, the TRPC3 inhibitor Pyr3 was applied to LN229 and U87 MG and GBM cell lines for in vivo and in vitro study. The survival rates for LN229 and U87 MG cells were decreased after $5 \mu \mathrm{M}$ Pyr3 treatment for $24 \mathrm{hrs}$ (Fig. 1C). In addition, the mitochondrial membrane potential (MMP) of LN229 cells was measured by JC-1 staining (Fig. 1D). The ratio of red to green fluorescence was significantly lower after $3 \mu \mathrm{M}$ Pyr3 treatment for $24 \mathrm{hrs}$ than after control treatment. These results suggest that TRPC3 inhibition impaired MMP stability and decreased the survival rate in GBM cells.

\section{Pyr3 inhibits migration and invasion in GBM cells}

After LN229 and U87 MG cells were treated with $1 \mu \mathrm{M}$ Pyr3, their cell migration ability was reduced to $60 \%$ of that of the control cells. Treatment with $1.5 \mu \mathrm{M}$ Pyr3 reduced the cell migration ability to $40 \%$ of that of the control group (Fig. 2A and 2B). Invasion was decreased by $30 \%$ after $1 \mu \mathrm{M}$ Pyr3 treatment and by $80 \%$ after $1.5 \mu \mathrm{M}$ Pyr3 treatment in LN229 cells (Fig. 2C and 2D). Invasion in U87 MG cells was decreased by $40 \%$ after $1 \mu \mathrm{M}$ Pyr3 treatment and by $70 \%$ after $1.5 \mu \mathrm{M}$ Pyr3 treatment (Fig. 2C and 2D). These results indicate that cell migration and invasion were inhibited by Pyr3 in GBM cells.

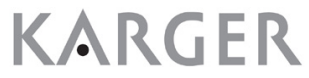


Fig. 1. TRPC3 is correlated with survival in GBM patients and cell lines. (A) Up-regulation of TRPC3 mRNA expression decreases survival times in human high-grade glioma patients. A KaplanMeier survival curve shows shorter survival times in patients with high TRPC3 expression levels $(n=20)$ than in those with low TRPC3 expression levels $(\mathrm{n}=6)(\mathrm{P}=0.0215$ by log -rank test, hazard ratio: 2.629 , 95\% CI: 1.370-17.30). (B) TRPC3 expression in normal human astrocytes (HAs) and LN229, U87MG and GBM8401 cells. The lower panels show the quantitative analyses of TRPC3 expression. ${ }^{* *} \mathrm{p}<0.001$ compared with the HA group. (C) LN229 and U87MG glioma cells were treated with different concentrations of Pyr3 $(1,1.5,3,5,10,20$ or $40 \mu \mathrm{M})$ for 24 hours. The survival rates were analyzed by SRB assay. ${ }^{*} \mathrm{p}<0.05$; ${ }^{* * *} \mathrm{p}<0.001$ compared with the control group. (D) LN229 glioma cells were treated with different concentrations of Pyr3 $(3,5$, or $10 \mu \mathrm{M})$ for 24 hours. JC-1 staining was used to analyze the mitochondria membrane potential by flow cytometry. The right panels show the quantitative analyses of the JC-1 red/green fluorescence ratios. ${ }^{*} \mathrm{p}<0.05 ;{ }^{* * *} \mathrm{p}<0.001$ compared with the control group.
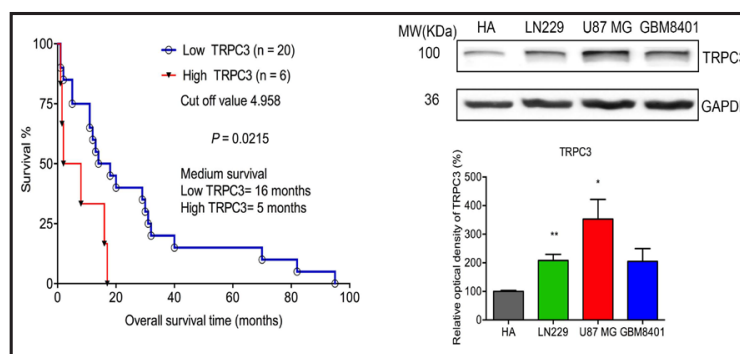

(A)

(B)
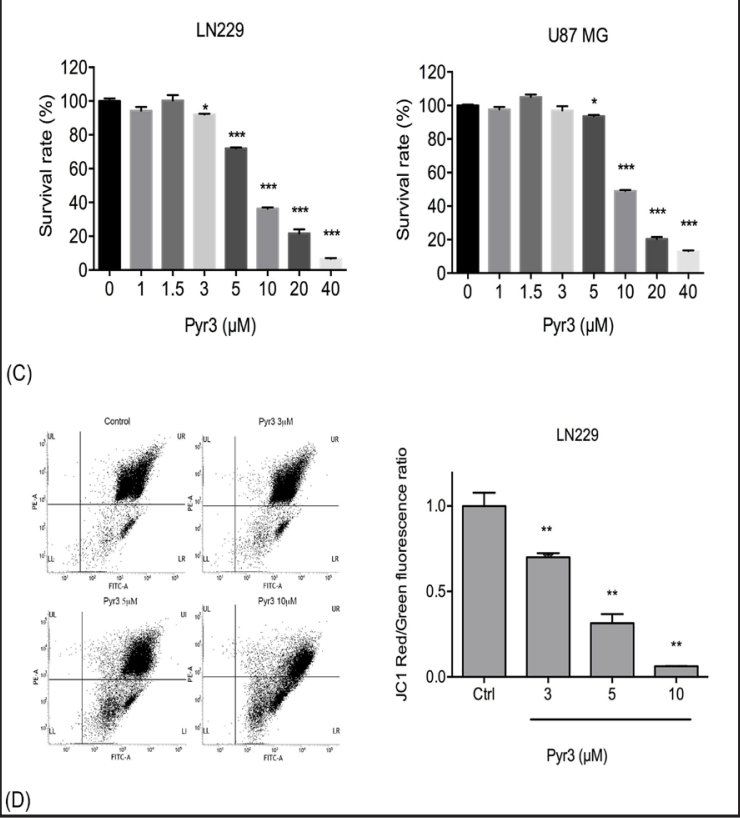

Fig. 2. Pyr3 induces glioma cell apoptosis. LN229 cells were treated with Pyr3 $(3,5$, or $10 \mu \mathrm{M})$ for 24 hrs and then stained with PI (A) or PE Annexin $\mathrm{V}$ Apoptosis and 7-AAD (B). The lower panels show the quantitative analyses of the percentages of early apoptotic cells. ${ }^{*} \mathrm{p}<0.05 ;{ }^{* *} \mathrm{p}<0.01$; $* * * \mathrm{p}<0.001$ compared with the control group.

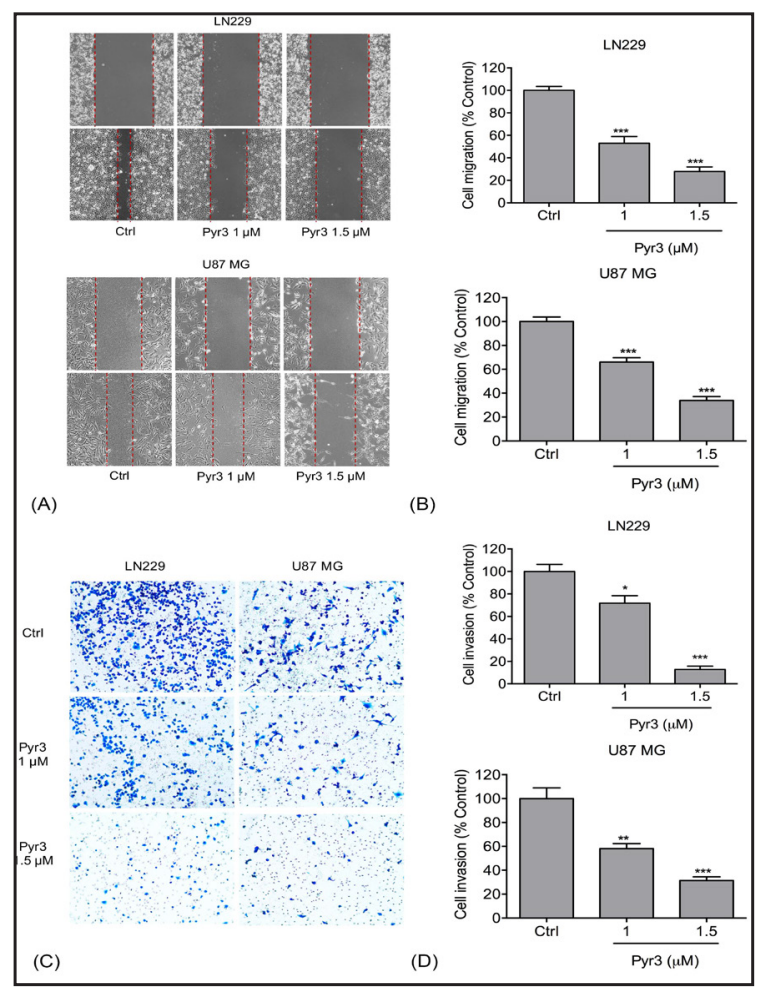




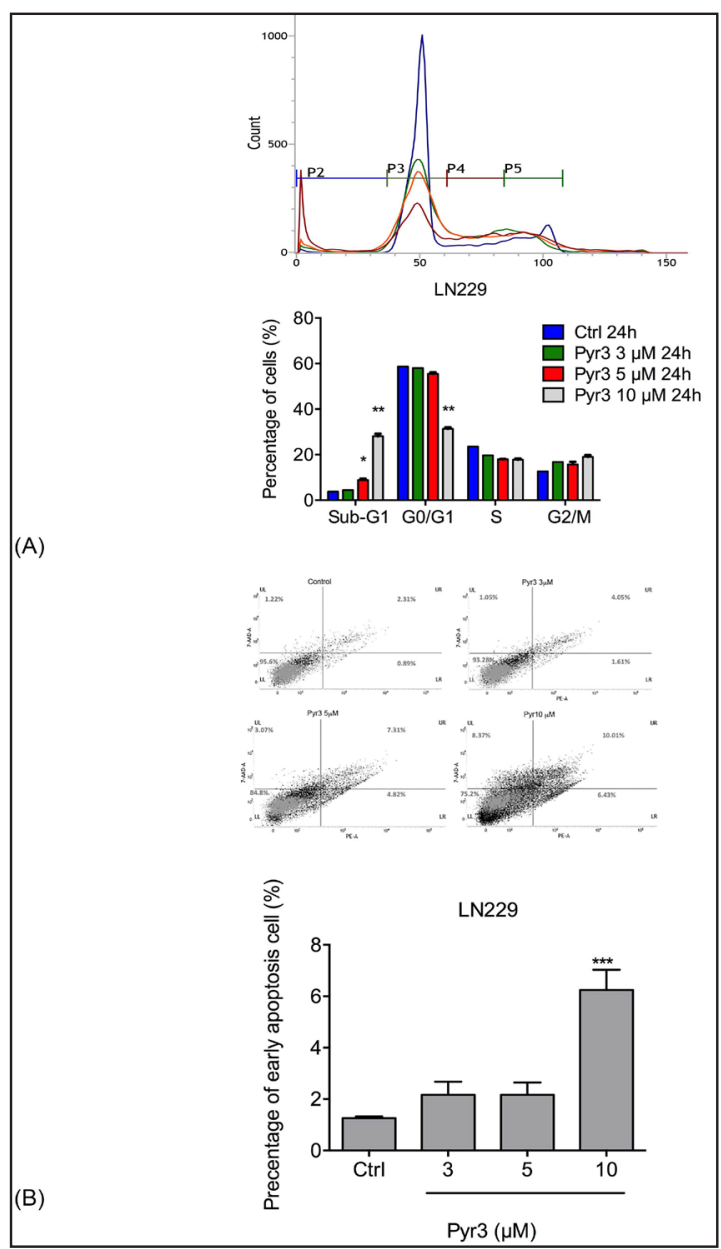

Fig. 3. Pyr3 inhibits cell migration and invasion in glioma cells. The inhibition of migration and invasion by Pyr3 in LN229 and U87MG cells was analyzed using (A) wound healing migration assays and (B) Transwell migration assays. The right panels show the quantitative analyses of the wound healing and Transwell migration assays. ${ }^{*} \mathrm{p}<0.05 ;{ }^{* * *} \mathrm{p}<0.001$ compared with the control group.

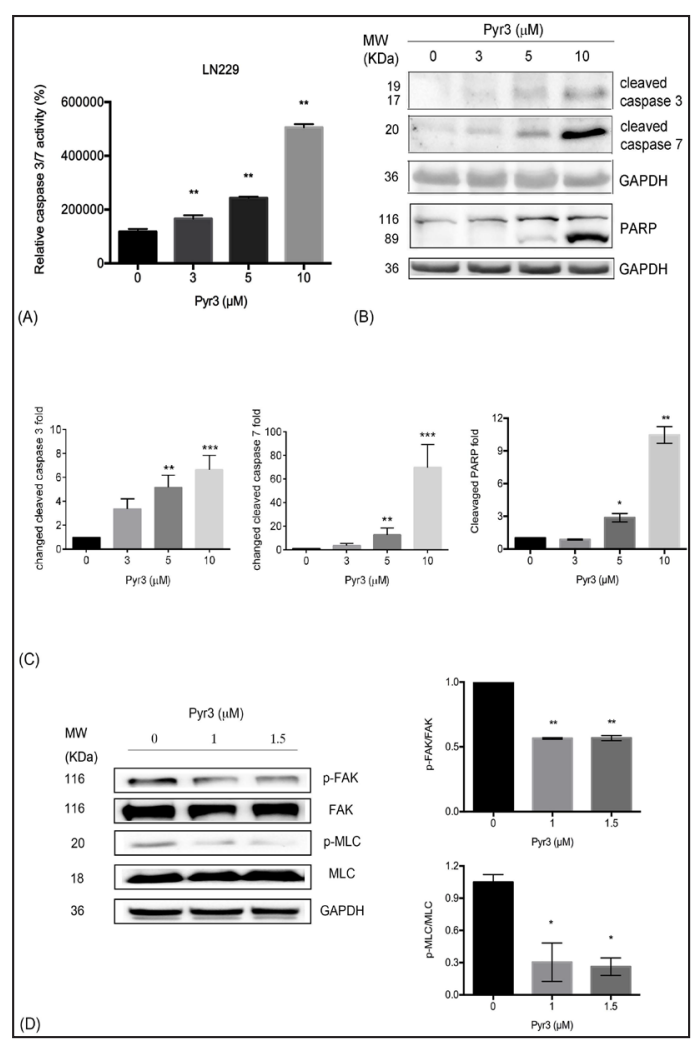

Fig. 4. Pyr3 induces caspase activation and focal adhesion protein dephosphorylation in LN229 cells. (A) After treating LN229 cells with Pyr3 $(3,5$, or $10 \mu \mathrm{M})$, caspase $3 / 7$ activity was analyzed by GLO. (B) Cleaved caspase 3, caspase 7, and PARP was analyzed by Western blotting. GAPDH was used as an internal control. ${ }^{*} \mathrm{p}<0.05$; ${ }^{* *} \mathrm{p}<0.01$ compared with the control group. (C) The quantitative analyses of the fold changes for cleaved caspase 3, caspase 7, and PARP. (D) After treating LN229 cells with Pyr3 (1 or $1.5 \mu \mathrm{M})$, the focal adhesion proteins p-FAK, FAK, p-MLC, and MLC were analyzed by Western blotting. GAPDH was used as an internal control. ${ }^{*} \mathrm{p}<0.05 ;{ }^{* *} \mathrm{p}<0.01$ compared with the control group. The right panels show the quantitative analyses of the p-FAK/FAK and p-MLC/MLC ratios.

\section{Pyr3 induces apoptosis in GBM cells}

Next, the effects of Pyr3 on the cell cycle and apoptosis were further investigated. The sub-G1 population was increased by 2- and 7-fold after $5 \mu \mathrm{M}$ and $10 \mu \mathrm{M}$ Pyr3 treatment, respectively, for 24 hrs compared to control treatment in LN229 cells (Fig. 3A). In addition, LN299 cells were stained with Annexin V-PE and 7-AAD after Pyr3 treatment. The percentage of apoptotic cells was increased from $2.31 \%$ to $10.01 \%$, indicating Pyr3-induced apoptosis in GBM cells (Fig. 3B). Moreover, caspase 3/7 activity, cleaved caspase 3, cleaved caspase 7, and PARP cleavage were increased by $5 \mu \mathrm{M}$ and $10 \mu \mathrm{M}$ Pyr3 treatment (Fig. 4A-C). These results show that Pyr3 increased the cell population in the sub-G1 phase and induced caspasedependent apoptosis in LN229 glioma cells. 
Pyr3 impairs focal adhesion and cytoskeleton protein expression in LN229 cells

The mechanisms of Pyr3induced reductions in migration and invasion were further studied by analyzing the expression of FAK and MLC. The expression levels of p-FAK and p-MLC were significantly reduced in LN229 glioma cells after Pyr3 treatment (Fig. 4C). These results suggest that Pyr3 inhibited migration and invasion through inactivating FAK and MLC.

\section{Pyr3 inhibits glioma tumor growth in a xenograft orthotopic model}

To further analyze the effects of Pyr3 in vivo, LN229-Luc2 cells were implanted in nude mice. Four days after implantation, the mice were administered Pyr3 alone or Pyr3 combined with temozolomide (TMZ). The tumor sizes were significantly decreased by TMZ and Pyr3 treatment compared to placebo treatment (Fig. 5A and 5C). There were no significant changes in body weight for any group after drug treatment (Fig. 5B). These results suggest that Pyr3 inhibits glioma tumor growth in vivo.

\section{Discussion}

Several reports show that cancer cell proliferation can be inhibited by blocking TRPC channels. TRPC1, TRPM7 and TRPM8 expression levels are correlated with breast cancer proliferation [10]. TRPC6 inhibition reduces proliferation in non-small cell lung cancer cells (NSCLCs) [11]. Proliferation in melanoma cells is also inhibited by Pyr3 treatment via reductions in pAKT and pSTAT5 levels [5]. Wang et al. show that TRPC3 knockdown and Pyr3 treatment decrease air way smooth muscle cell proliferation in ovalbumin-sensitized mice [12]. Our findings also suggest that Pyr3 inhibits glioma cell proliferation.

A previous study showed that TRPC1 inhibits migration through decreasing the sphingosine1-phosphate 3 receptor and vascular endothelial growth factor receptor 2 levels in thyroid cancer cells [13]. In NSCLCs, TRPC6 inhibition suppresses the expression of fibronectin and the tight junction protein zonula occluden protein-1 (ZO-1), leading to a decrease in migration ability [11]. In Balb/c nu/nu mice, wound contraction was enhanced by transplanting TRPC3-overexpressing fibroblasts [14]. In addition, TRPC3 knockdown 
inhibits the migration of olfactory ensheathing cells [15]. In melanoma cells, migration is also inhibited by Pyr3 [5]. In breast cancer cells, migration and invasion are inhibited by silencing TRPV6 [10]. In our study, migration and invasion abilities were inhibited by Pyr3 via decreased FAK and MLC dephosphorization in glioma cells. Pyr3 inhibits glioma cell migration through impairing focal complex formation and cytoskeleton imbalance.

Pyr3 has been reported to induce apoptosis in melanoma cells [5]. Pyr3 enhances Dex-induced apoptosis by increasing MMP depolarization in acute lymphoblastic leukemia cells [16]. Another TRPC blocker, SK\&F96365, has been reported to induce apoptosis by increasing caspase-3/9 activity and MMP depolarization in A7r5 smooth muscle cells [17]. TRPC3-overexpressing fibroblasts induce NF- $\kappa B$ activation via $\mathrm{Ca}^{2+}$ influx [14]. Therefore, Pyr3 may induce cell death and apoptosis through $\mathrm{Ca}^{2+}$ imbalance in the cells. Although Pyr3 has been reported to inhibit melanoma cell proliferation and migration, it has not been used to treat glioma tumors in vitro or in vivo. This is the first study to show that Pyr3 inhibits not only glioma tumor growth, migration and invasion in vitro but also glioma tumor growth in a xenograft orthotopic model. Pyr3-TMZ combination therapy could be used to treat glioblastoma in the future.

\section{Acknowledgements}

The authors would like to thank the Dr. Dueng-Yuan Hueng, National Defense Medical Center, Taiwan, for kindly provided the human LN229 and U87MG glioblastoma cell lines and GBM8401 primary cell line. This work was supported by the Ministry of Science and Technology, Taiwan (grant number: 107-2320-B-016-011-MY3) and the Zuoying Branch of Kaohsiung Armed Forces General Hospital (ZBH 107-07).

\section{Disclosure Statement}

The authors declare no conflicts of interest for this article.

\section{References}

1 Paw I, Carpenter RC, Watabe K, Debinski W, Lo H-W: Mechanisms regulating glioma invasion. Cancer Lett 2015;362:1-7.

2 Safa AR, Saadatzadeh MR, Cohen-Gadol AA, Pollok KE, Bijangi-Vishehsaraei K: Emerging targets for glioblastoma stem cell therapy. J Biomed Res 2015;30.

-3 Wen PY, Lee EQ, Reardon DA, Ligon KL, Alfred Yung WK: Current clinical development of PI3K pathway inhibitors in glioblastoma. Neuro Oncol 2012;14:819-829.

4 Oike T, Suzuki Y, Sugawara K, Shirai K, Noda SE, Tamaki T, Nagaishi M, Yokoo H, Nakazato Y, Nakano T: Radiotherapy plus concomitant adjuvant temozolomide for glioblastoma: Japanese mono-institutional results. PLoS One 2013;8:e78943.

5 Oda K, Umemura M, Nakakaji R, Tanaka R, Sato I, Nagasako A, Oyamada C, Baljinnyam E, Katsumata M, Xie LH, Narikawa M, Yamaguchi Y, Akimoto T, Ohtake M, Fujita T, Yokoyama U, Iwatsubo K, Aihara M, Ishikawa Y: Transient receptor potential cation 3 channel regulates melanoma proliferation and migration. J Physiol Sci 2017;67:497-505.

6 Aydar E, Yeo S, Djamgoz M, Palmer C: Abnormal expression, localization and interaction of canonical transient receptor potential ion channels in human breast cancer cell lines and tissues: a potential target for breast cancer diagnosis and therapy. Cancer Cell Int 2009;9:23.

7 Yang SL, Cao Q, Zhou KC, Feng YJ, Wang YZ: Transient receptor potential channel C3 contributes to the progression of human ovarian cancer. Oncogene 2009;28:1320-1328.

-8 Bomben VC, Turner KL, Barclay TT, Sontheimer H: Transient receptor potential canonical channels are essential for chemotactic migration of human malignant gliomas. J Cell Physiol 2011;226:1879-1888. 


\section{Cellular Physiology Cell Physiol Biochem 2018;48:1694-1702

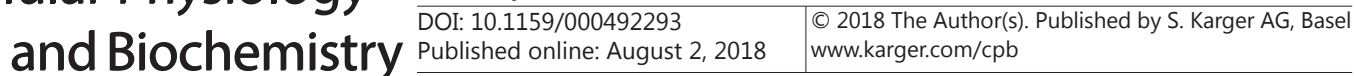

-9 Uemura T, Green M, Warsh JJ: Chronic LiCl pretreatment suppresses thrombin-stimulated intracellular calcium mobilization through TRPC3 in astroglioma cells. Bipolar Disord 2016;18:549-562.

10 Dhennin-Duthille I, Gautier M, Faouzi M, Guilbert A, Brevet M, Vaudry D, Ahidouch A, Sevestre H, OuadidAhidouch H: High Expression of Transient Receptor Potential Channels in Human Breast Cancer Epithelial Cells and Tissues Correlation with Pathological Parameters. Cell Physiol Biochem 2011;28:813-822.

11 ang LL, Liu BC, Lu XY, Yan Y, Zhai YJ, Bao Q, Doetsch PW, Deng X, Thai TL, Alli AA, Eaton DC, Shen BZ, Ma HP: Inhibition of TRPC6 reduces non-small cell lung cancer cell proliferation and invasion. Oncotarget 2015;8:5123-5134.

12 Wang L, Li J, Zhang J, He Q Weng X, Huang Y, Guan M, Qiu C: Inhibition of TRPC3 downregulates airway hyperresponsiveness, remodeling of OVA-sensitized mouse. Biochem Biophys Res Commun 2017;484:209217.

-13 Asghar MY, Magnusson M, Kemppainen K, Sukumaran P, Lof C, Pulli I, Kalhori V, Tornquist K: Transient Receptor Potential Canonical 1 (TRPC1) Channels as Regulators of Sphingolipid and VEGF Receptor Expression: IMPLICATIONS FOR THYROID CANCER CELL MIGRATION AND PROLIFERATION. J Biol Chem 2015;290:16116-16131.

14 Ishise H, Larson B, Hirata Y, Fujiwara T, Nishimoto S, Kubo T, Matsuda K, Kanazawa S, Sotsuka Y, Fujita $\mathrm{K}$, Kakibuchi M, Kawai K: Hypertrophic scar contracture is mediated by the TRPC3 mechanical force transducer via NFkB activation. Sci Rep 2015;5:11620.

15 Wang Y, Teng HL, Gao Y, Zhang F, Ding YQ Huang ZH: Brain-derived Neurotrophic Factor Promotes the Migration of Olfactory Ensheathing Cells Through TRPC Channels. Glia 2016;64:2154-2165.

-16 Abdoul-Azize S, Buquet C, Vannier JP, Dubus I: Pyr3, a TRPC3 channel blocker, potentiates dexamethasone sensitivity and apoptosis in acute lymphoblastic leukemia cells by disturbing $\mathrm{Ca}(2+)$ signaling, mitochondrial membrane potential changes and reactive oxygen species production. Eur J Pharmacol 2016;784:90-98.

17 Park EJ, Kim SY, Kim SH, Lee CR, Kim IS, Park JK, Lee SW, Kim BJ, Chun JN, So I, Jeon JH: SK\&F 96365 induces apoptosis and autophagy by inhibiting Akt-mTOR signaling in A7r5 cells. Biochim Biophys Acta 2011;1813:2157-2164. 